\title{
Contextos adversos para la participación: intercambio y consumo de drogas. Resistencias en barrios populares del Gran San Miguel de Tucumán, Argentina
}

\section{Adverse contexts for participation: drug exchange and consumption. Resistance in popular neighborhoods of Greater San Miguel de Tucumán, Argentina}

\author{
Debora Decima ${ }^{1}$ y Paula Boldrini ${ }^{2}$ \\ Recibido: 15/10/20. Aceptado: 05/04/21 \\ http://dx.doi.org/10.30972/dpd.10154810
}

\section{Resumen}

El artículo presenta un estudio de experiencias participativas en uno de los barrios populares paradigmáticos del Gran San Miguel de Tucumán, ciudad intermedia, cabecera del Noroeste Argentino. El barrio Costanera Norte es foco de una multiplicidad de políticas públicas debido a los elevados niveles de pobreza y vulnerabilidad que caracterizan a esta población.

Es en este contexto y, a su vez, en uno de los grupos más vulnerables dentro del barrio, como lo son familias y jóvenes afectados por problemas derivados de la venta y el consumo de drogas, que se llevan adelante experiencias de participación que tienen al hábitat y la comunicación como ejes convocantes.

La investigación busca caracterizar y analizar procesos de participación barrial, a partir del seguimiento de dos experiencias, abordadas desde la observación participante de escenas y la realización de entrevistas. Los resultados permiten reelaborar el desarrollo de cada uno de los aspectos estructurantes de los procesos participativos en contextos particularmente adversos, promoviendo la salud a partir del trabajo grupal y colectivo sobre emergentes sociales elegidos, reformulados y desplegados por la propia comunidad.

Palabras clave: participación; drogas; barrios populares.

\footnotetext{
${ }^{1}$ Licenciada en Ciencias de la Comunicación (Universidad Nacional de Tucumán). Becaria Doctoral CONICET Actualmente cursando el Doctorado en Ciencias Sociales (orientación Geografía) (Universidad Nacional de Tucumán) - INSTITUTO DE INVESTIGACIONES TERRITORIALES Y TECNOLÓGICAS PARA LA PRODUCCIÓN DEL HÁBITAT (INTEPH, CONICET-UNT). Correo electrónico: decimaperiodista@gmail.com

${ }^{2}$ Arquitecta (Universidad Nacional de Tucumán) y doctora en Ciencias Sociales (orientación Geografía) (Universidad Nacional de Tucumán). Investigadora asistente del CONICET - INSTITUTO DE INVESTIGACIONES TERRITORIALES Y TECNOLÓGICAS PARA LA PRODUCCIÓN DEL HÁBITAT (INTEPH, CONICET-UNT). Correo electrónico: Paula boldrini@hormail.com
} 


\section{Abstract}

The article presents a participative experiences study in one of the paradigmatic popular suburbs of Gran San Miguel de Tucuman, top city of the Argentinian Northwest region. Costanera Norte suburb is a multiplicity of public politcs epicenter as a consequence of the high levels of poverty and vulnerability, typical for this population. Is in this context and at the same time in one of the most vulnerable groups, such as families and young residents affected by problems derived from the sale and consumption of drugs, where participative experiences take place, which have habitat and comunication as the convening subjects.

The research seeks for characterize and analyze suburb's participation processes from two experiences, adressed from participating observation of situations and the making of interviews. The results allow to reelaborate the development of each one of the structural aspects of the participative processes in particularly unfavorable contexts, promoting health from groupal and collective work about chosen, reformulated and carrried out by the community emerging social phenomena.

Keywords: participation, drugs, popular suburbs.

\section{Fundamentación y antecedentes}

En América Latina como también en Argentina, la problemática del intercambio y consumo de drogas se incrementa en la década de 1970 (Touzé, 2006), dando lugar a la promulgación de leyes -bajo la lógica prohibicionista- que promueven el aumento de penalidades. Bajo esta misma lógica se crea la Secretaría de Programación para la Prevención de la Drogadicción y la Lucha contra el Narcotráfico (Sedronar) en 1989, dependiente del gobierno nacional, encargada de articular medidas tanto punitivas como sanitarias.

El paso de los años no redundó en mejoras y según el estudio realizado por la Sedronar a través del observatorio argentino de drogas, el consumo de pasta base en 2010 y 2017 aumentó de 61168 a 99815 registros sobre población de 12 a 65 años. Esto representa un incremento del $66 \%$ en un lapso de siete años. Asimismo, en ambos casos se remarca que el uso de esta droga se concentra en jóvenes de entre 12 y 24 años, bajando cada vez más la edad de inicio en el consumo. En la provincia de Tucumán, según el informe local de 2017, esta problemática afecta principalmente a hombres y presenta una prevalencia de vida en aumento $^{3}$, a diferencia del registro resultante del resto de las sustancias en el contexto nacional (a nivel provincial fue de 1,9\% y a nivel nacional de 0,9\% en 2017).

Las instituciones públicas provinciales han dado respuesta generando espacios de registro e intervención sobre la problemática del consumo de drogas, sobre todo alrededor del

\footnotetext{
${ }^{3}$ La prevalencia de vida refiere a la proporción de personas que consumieron la sustancia alguna vez en la vida sobre el total de las personas estudiadas.
} 
intercambio y consumo de pasta base en barrios populares ${ }^{4}$. Una de las principales fuentes de información de este trabajo es el coordinador territorial de la Secretaría de Asistencia y Prevención de Adicciones del Ministerio de Desarrollo Social, que reconoce el aumento de jóvenes y niños en los circuitos de consumo e intercambio de drogas. Según su perspectiva, el mayor desafío se encuentra en el campo concreto de trabajo, en contacto directo con jóvenes que no buscan ayuda, sino que deben ser acompañados en el ámbito en el que viven y desarrollan su cotidianeidad.

En este contexto y en el marco de una multiplicidad de abordajes posibles sobre esta compleja problemática, los principales antecedentes considerados giran en torno a la participación de sectores populares bajo condiciones de extrema vulnerabilidad, afectados por la venta y el consumo de drogas como fenómeno particularmente crítico. Esta problemática se manifiesta en forma especialmente conflictiva entre la constelación de necesidades insatisfechas que detentan habitantes de asentamientos informales del Gran San Miguel de Tucumán (GSMT).

Las condiciones sociales en barrios populares han sufrido un retroceso cualitativo durante las últimas dos décadas fundamentalmente, derivado de la inserción y del avance acelerado de hábitos vinculados a la venta y el consumo de drogas como la pasta base de cocaína. También llamada paco en Argentina, la pasta base es cocaína fumable y constituye uno de los pasos intermedios en el proceso de la obtención del clorhidrato de cocaína a partir de las hojas de coca. Es un producto con menos pureza que la cocaína, por lo tanto, más fácil de elaborar y que los narcotraficantes pueden producir más rápido y vender más barato (Arrieta, Prieto y Damin, 2020). Este fenómeno está asociado a la pobreza tanto para explicar su consumo como a la red de conductas cotidianas que se entrelazan para dar lugar a la consolidación de nuevas dinámicas barriales en torno a este problema (Boldrini, 2019). Es en esta cotidianeidad que se entrecruzan los tiempos y espacios destinados a la familia, el trabajo y el tiempo libre (Quiroga y Racedo, 1995) de manera particular, estableciendo nuevas lógicas de comunicación y producción del hábitat, entre otras. Tanto en el abordaje de la salud para analizar el consumo de sustancias psicoactivas, como en la comunicación y el hábitat, se presentan paradigmas enfrentados que responden a diferentes modelos e intereses. En todos estos modelos se contraponen concepciones de participación disímiles. Salud, comunicación y hábitat serán analizados a partir de una perspectiva atravesada por la

\footnotetext{
${ }^{4}$ Es el Ministerio de Desarrollo Social (MDS) quien lleva adelante una política territorial específica a través de la Secretaría de Prevención y Asistencia de las Adicciones, que promueve, planifica, evalúa y coordina acciones referidas a la problemática del consumo de drogas, desde una lógica social, global y policausal. Está organizada a partir de tres direcciones: 1. Prevención, a cargo de los Centros Preventivos Locales de Adicciones (Cepla), 2. Asistencia, a cargo de los Dispositivos de Salud en Adicciones (DSA), y 3. Observatorio, a cargo del Observatorio Provincial de Drogas. Tanto los Cepla como los DSA tienen un funcionamiento de carácter territorial. Los primeros operan de forma nodal, con seis sedes en el GSMT, abocadas al buen uso del tiempo libre para los vecinos, enfocándose además en el acompañamiento, cuidado y tratamiento ambulatorio a personas con consumo problemático de sustancias.
} 
participación, entendida como mecanismo en el que prevalecen relaciones dialógicas e integración comunitaria, a partir de tareas compartidas destinadas a resolver necesidades priorizadas por los propios destinatarios y resueltas por medios también elegidos y llevados adelante por ellos, en articulación con otros actores que comparten, respetan y promueven los ámbitos y tiempos propios de este tipo de procesos.

\section{Intercambio y consumo de drogas, salud y modelos de políticas diferenciados}

La pasta base de cocaína representa una droga que ocasiona un acelerado deterioro físico y mental, así como un elevado nivel de dependencia que impacta en la estructura social mediante la descomposición de vínculos familiares y comunitarios, la profundización de la segregación social y el aumento de la violencia (Boldrini, 2019; Trufo et al., 2017).

En el campo de las políticas que contrarrestan el consumo y comercialización de drogas, se enfrentan el paradigma prohibicionista con el de reducción de daños. Estos modelos plantean diferentes modos de concebir, planificar e intervenir en el territorio mediante políticas públicas específicas. El modelo dominante conocido como prohibicionista, con un marcado sesgo capitalista (Touzé, 2014), asume en un mismo nivel y calidad tanto a productores y distribuidores como a consumidores, lo que explica la prevalencia de acciones represivas (Llovera y Scialla, 2017). Este enfoque de tipo punitivo se asocia a la promoción de la salud mental desde una perspectiva manicomial, orientada al tratamiento individual basado en la internación. En disputa se encuentra el modelo de reducción de daños, que diferencia promotores del intercambio de aquellos que consumen. A estos últimos los considera desde una perspectiva de salud mental y comunitaria ampliada, concebida desde una política integral (Llovera y Scialla, 2016). La salud en este caso es entendida como capacidad de adaptarse en forma activa, es decir, sin perder la posibilidad de transformar la realidad y al propio sujeto en las relaciones que lo determinan (Quiroga, 2005). Este modelo implica un conjunto de estrategias orientadas a reducir las consecuencias negativas de ciertos comportamientos humanos que, en el ámbito específico de las drogas, entiende al consumo como una conducta compleja, multifacética y que forma parte de la sociedad, por lo que la implementación de políticas orientadas a este modelo demanda tener en cuenta las circunstancias tanto individuales como comunitarias, de cada región donde se apliquen. Enmarcada en el enfoque de la salud pública, la prevención comunitaria permite también disminuir los impactos sanitarios y sociales de aquellas poblaciones que no padecen problemas de consumo o no se interesan en la abstención, por lo que las estrategias se centran en la educación de los usuarios de drogas y en la generación de marcos legales que faciliten dichos procesos (Damin y Arrieta, 2020). Este modelo básicamente cuida la salud de aquellos que deciden consumir una sustancia y los que no pueden dejar de hacerlo, así como la salud de su entorno. 
Este segundo paradigma es el modelo declarado desde el Ministerio de Desarrollo Social (MDS) de la provincia de Tucumán. No obstante, presenta serias contradicciones entre discursos, concepciones y acciones concretas, en las que se evidencian persistencias asociadas a la lógica prohibicionista en disputa con lo nuevo, referido a la adhesión al modelo de reducción de daños (Boldrini, 2019). Del MDS depende la Secretaría de Prevención y Asistencia de las Adicciones, que promueve, planifica, evalúa y coordina acciones referidas a la problemática adictiva en diferentes ámbitos. En el ámbito barrial se despliega la interacción concreta con los sujetos adictos y su entorno, por lo que se aborda a través de la acción de los Centros Preventivos Locales de las Adicciones (Cepla), también llamados Centros Educativos Terapéuticos (CET). Estos centros funcionan en seis barrios populares ${ }^{5}$, uno de ellos, Costanera. A esto se suman los Dispositivos de Salud en Adicciones (DSA), que habilitan la experiencia sujeta a estudio en esta investigación. Son los DSA, existentes en solo dos barrios populares, los que operan directamente sobre los vínculos barriales, inmersos y enfrentados a los procesos de producción y comercialización de sustancias a menor escala, conocidos en los barrios populares de Argentina como narcomenudeo. Estos vínculos están atravesados -entre otros aspectos- por los modos dominantes de: a. producción del hábitat, y b. comunicación en los barrios populares.

\section{a. La producción del hábitat popular}

Este circuito marcado por la producción, intercambio y consumo de drogas en los barrios configura nuevos hábitos y costumbres individuales y colectivas que se evidencian en el ámbito socio-espacial. La venta se instala en el barrio, desde la casa de algún vecino conocido como dealer, sumado a jóvenes - principalmente varones- que tienden a reunirse en grupo en las esquinas o en espacios públicos -plazas, canchas- para escuchar música, beber y consumir drogas. Vendedores de drogas se refieren a ellos como soldaditos, usados para efectuar la comercialización de las sustancias y disputar el territorio con otros dealers del barrio (Del Frade, 2014).

La cotidianeidad se cristaliza de manera tangible e intangible en el espacio vivido de manera rutinaria, mediante una red de relaciones conflictiva cuya principal evidencia se expresa en la colonización nociva del espacio público. Es allí donde se dirimen los conflictos comunitarios de diferentes características tales como el intercambio de drogas, consumo, peleas y suicidios. El espacio público se torna peligroso, en un ámbito donde el espacio familiar resulta insuficiente, con condiciones de salubridad y capacidad de contención deficitaria en la mayoría de las viviendas de estos barrios. Según Borja y Muxi (2000), el espacio público es donde el carácter de la sociedad se hace visible, a través de las relaciones

\footnotetext{
${ }^{5}$ En el Gran San Miguel de Tucumán, el Registro Nacional de Barrios Populares (Renabap) apuntó hasta 2016 más de 200 barrios populares. No obstante, ya el Instituto de Vivienda provincial contaba más de 300 en registros propios anteriores, sin que hubiera avances significativos en materia de mejora de estos barrios.
} 
entre los habitantes, el poder y la ciudadanía. Por eso, resulta central comprender la magnitud y el carácter de que hoy sea la violencia el aspecto que lo caracterice.

En diferentes formas, de acuerdo a su tipo o localización dentro de la ciudad, este espacio ejerce un rol de indicador de déficit democrático al momento de tomar decisiones sobre su hábitat. En sectores vulnerables en particular, la violencia se acentúa con la criminalización de grupos y territorios designándolos peligrosos y sospechosos colectivamente. Esto se expresa con el sostenimiento de políticas represivas y la incomprensión de la cuestión urbana en todas sus dimensiones, tales como la fragmentación de las periferias, la falta de infraestructura y equipamiento, escasez de cualificación del espacio público -cuando lo hayy el ejercicio de dinámicas sociales particulares, habitualmente estigmatizadas. La violencia e inseguridad debe ser entendida como señal de alerta social, la evidencia de exclusión y desintegración social en todos sus aspectos por parte de sectores que viven en la ciudad sin formar parte a ella y que expresan su malestar a través de señales violentas (Borja y Muxi, 2000).

\section{b. Los procesos de comunicación}

La comunicación, entendida como proceso de producción, circulación y uso del sentido social, interviene significativamente en los modos de satisfacción de necesidades para la supervivencia de los sujetos, en el marco de procesos simbólicos en constante relación con la realidad material. Es en el escenario de la vida cotidiana donde sujeto y comunidad se configuran, en el marco de las relaciones que establecen entre ellos y con su contexto (Quiroga, 2001). De las relaciones sociales que gestan ese orden, las instituciones y las prácticas que expresan esas relaciones, emergen las formas de pensamiento socialmente compartido. Allí donde se evidencia la producción y reproducción de la ideología dominante, que se presenta ante los sujetos como dada, naturalizada y reforzada por los medios de comunicación hegemónicos, cuya tarea consiste en exaltar unos aspectos y ocultar otros, en beneficio de los sectores de poder (Vinelli, 2014). Este fenómeno se manifiesta en las formas de silenciamiento y estigmatización de ideas antagónicas, así como en la descontextualización intencionada de noticias con el propósito de desviar la comprensión de las circunstancias en que ciertos hechos suceden (De Moraes, 2013).

La producción y reproducción de tales discursos en ocasiones cumple su cometido, vaciando de análisis críticos y expresiones de disenso, evitando roces entre las interpretaciones de los hechos y su entendimiento, por parte de individuos, grupos y clases. Dificultando tanto la articulación entre sectores sociales como la integración en el seno de las comunidades, reforzando el fenómeno de exclusión mediante la imposibilidad de participación en los discursos de los medios de comunicación de masas.

Los modos en que decidimos ver e interpretar la realidad son el resultado de una construcción social. En un proceso de radicalización del sentido común en el que se 
presentan constantemente como certezas ciertos hechos de la realidad, obviando que la mirada, interpretación y significación es construida dentro de un contexto (Grimson, 2011). Significamos y al hacerlo, identificamos a nosotros, a la vez que a otros. Esas formas de ver, interpretar y significar se convierten en prácticas sociales, objetos materiales y simbólicos se tipifican y a partir de allí las sociedades se organizan. Por lo tanto, los barrios populares deben ser entendidos -estudiados- tomando en cuenta las relaciones de poder. En toda relación social hay circulación de poder y en toda configuración el poder adquiere las peculiaridades de la hegemonía, de la producción de sentidos comunes y la naturalización de lo subalterno. Sin embargo, la hegemonía debe ser entendida como el establecimiento de un campo de posibilidades para el conflicto. Toda sociedad posee un conjunto de herramientas identitarias y clasificaciones, en un contexto histórico específico, que permiten a sus miembros identificarse a sí mismos e identificar a otros, dando lugar a sentidos positivos, a la vez que negativos, que sustentan la estigmatización. De allí que la disputa acerca de los sentidos, del conjunto de clasificaciones, resulte parte decisiva de los conflictos sociales (Grimson, 2011). Es por eso que la comunicación alternativa se presenta como estrategia para la democratización de la comunicación desde el barrio, a fin de cuestionar las verdades discursivas de los medios como aparatos privados de la hegemonía, desde espacios más libres de información y opinión (De Moraes, 2011). Los procesos de comunicación en el territorio agudizan una relación dialéctica donde la hegemonía comunicacional puede ser reelaborada en un proceso de resistencias y conquistas, en las que la defensa por la autoría de la palabra (Requejo, 2009) y la promoción de una comunicación democrática resulta imprescindible.

\section{Abordaje teórico-metodológico}

Con el encuadre antes desarrollado, proponemos reelaborar experiencias en las que se busca la participación social, en un barrio popular paradigmático del área metropolitana de Tucumán conocido como Costanera Norte.

Para el análisis de las experiencias, se asume a la participación como proceso -tangible e intangible- llevado adelante por un grupo o comunidad a partir de su propia iniciativa, en un ejercicio activo y sostenido en el tiempo, mediante ámbitos y prácticas apropiadas y transformadoras, a través de las cuales se construyen vínculos interpersonales capaces de mantener el predominio de la integración comunitaria (Boldrini, 2012). El factor intangible que se señala es el aspecto menos considerado en los programas y proyectos de mejora del hábitat. En su lugar, se tiende a suponer que la solución material otorga, por defecto, satisfacción a las necesidades. Este mecanismo pasa por alto la compleja constelación de necesidades que giran en torno a cada demanda. Es por eso que tener en cuenta aspectos inmateriales como las redes de relaciones sociales comunitarias, las experiencias vividas tanto individuales como colectivas que constituyen la identidad comunitaria a lo largo del 
tiempo y los complejos procesos comunicacionales sobre los que se constituyen se convierten en variables imprescindibles al momento de pensar políticas públicas apropiadas, respetuosas de las especificidades de cada grupo humano en su relación particular e irrepetible con su hábitat.

Estos aspectos permiten establecer las siguientes categorías de análisis: A. La secuencia histórica de la participación, que contiene fases de cada proceso particular y se encuentra inscripta en un proceso general; B. Los vínculos interpersonales y la integración comunitaria; y C. Los ámbitos y la modalidad de trabajo.

Para su abordaje, se procede a la presentación del caso donde transcurrieron las experiencias en estudio: la radio comunitaria entre 2012 y 2013 y la mejora del espacio público entre 2016 y 2017. Se prioriza la utilización de herramientas cualitativas, donde la observación participante (Yuni y Urbano, 2003; Guber, 2009; Taylor y Bogdan, 1990) constituye el eje vertebrador del trabajo de campo sobre distintas actividades en terreno tales como talleres, reuniones grupales, asambleas comunitarias y celebraciones de las que participaron los habitantes del barrio. Tanto la participación en dichas experiencias como la tarea de relevamiento fueron registradas a partir de las notas de campo, grabaciones, fotografías y material audiovisual (Vasilachis de Gialdino, 2015). En el sentido que plantean autores como Hammersley y Atkinson (1994), se considera el aporte de la etnografía como método de investigación social que trabaja con una amplia gama de fuentes de información. Entre las fuentes utilizadas se encuentra la realización de 10 entrevistas semiestructuradas (Mercadé, 1986; Taylor y Bogdan, 1984) a diferentes actores intervinientes en cada experiencia (vecinos, técnicos territoriales, funcionarios provinciales), analizadas mediante el método comparativo constante (Glaser y Strauss, 1967). Las consultas estuvieron orientadas a conocer las principales variables inherentes a la participación antes mencionadas, sobre los procesos de comunicación popular y mejora del hábitat colectivo.

\section{Costanera Norte y las experiencias de hacer radio y la plaza}

El Gran San Miguel de Tucumán (GSMT), una ciudad intermedia de casi 900000 habitantes, es cabecera de la región más pobre de la Argentina (Bolsi y Paolasso, 2009). La ciudad, en sintonía con los fenómenos urbanos latinoamericanos, creció de manera acelerada y desigual, dominada por el mercado inmobiliario configurando islas residenciales con tipologías residenciales homogéneas (Malizia, Boldrini, Paolasso, 2018). Dentro de cada isla, las familias residentes comparten una multiplicidad de características y problemáticas que, en el caso de los barrios populares, están condicionadas por la extrema vulnerabilidad.

La zona de la Costanera se encuentra ubicada al noreste del municipio capitalino (Figura 1), a las márgenes del río Salí, en el límite que separa a San Miguel de Tucumán del municipio Banda del Río Salí. Está situado sobre un área degradada, con condiciones de inundabilidad y contaminación por desagüe de canales pluviales y desechos emanados de 
fábricas y residuos cloacales -sin tratamiento previo- de algunos sectores de la ciudad (Del Castillo, 2012). Presenta una población mayormente perteneciente a sectores medio-bajos y bajos, la mayoría desocupada, dedicada principalmente a trabajos temporales como los que surgen en la cosecha del limón o la actividad del cartoneo, recolección de botellas o selección y acopio de otros residuos, lo que representa una estrategia intergeneracional de respuesta a las dificultades de inserción en el mercado de trabajo y a sus necesidades de reproducción social (Del Castillo, 2012).

Figura 1. Contexto urbano y localización del área de estudio

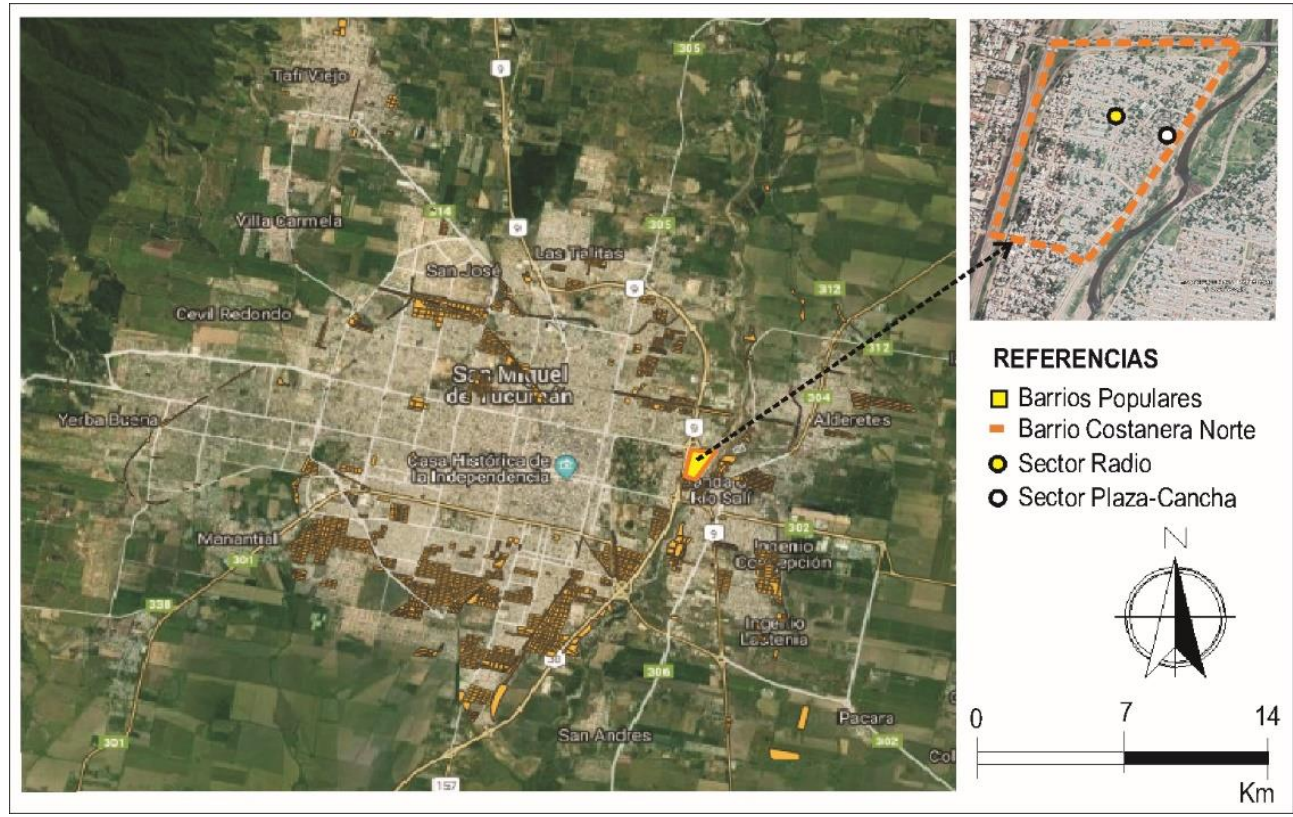

Fuente: elaboración propia a partir del trabajo de campo.

Los orígenes del barrio datan de la década de 1960 y se enmarcan en el proceso de expansión urbana de San Miguel de Tucumán que termina por configurar el aglomerado GSMT. Considerado villa miseria de acuerdo a su periodo de conformación (Cravino y Varela, 2008), creció gradualmente a partir del esfuerzo que los vecinos asumieron en la autoproducción del hábitat (Del Castillo, 2012). Hacia la década del 1980 incorporó una segunda oleada de pobladores, para extender el barrio hacia el sur y posteriormente hacia la ribera del río. Desde finales de la década del 1990 en adelante, comienza a instalarse en el barrio el consumo problemático ${ }^{6}$ de sustancias psicoactivas, siendo los inhalantes -

\footnotetext{
${ }^{6}$ La Ley Nacional 26.934 que establece el PLAN INTEGRAL PARA EL ABORDAJE DE LOS CONSUMOS PROBLEMÁTICOS, en su ARTíCULO $2^{\circ}$ expresa: "A los efectos de la presente ley, se entiende por consumos problemáticos aquellos consumos que -mediando o sin mediar sustancia alguna- afectan negativamente, en forma crónica, la salud física o psíquica del sujeto, y/o las relaciones sociales. Los consumos problemáticos pueden manifestarse como adicciones o abusos al alcohol, tabaco, drogas psicotrópicas -legales o ilegales- o producidos por ciertas conductas compulsivas de los sujetos hacia el juego, las nuevas tecnologías, la
} 
pegamento- la principal sustancia que consumían los jóvenes (Mustafá, 2016). Ya en 2006, a raíz del afianzamiento del narcotráfico en este y otros barrios, comienza a instalarse en Tucumán el consumo de pasta base de cocaína (Mustafá, 2016). Simultáneamente, el barrio comienza a experimentar algunas mejoras en materia de infraestructura a partir de la intervención del Estado, volviéndose un nodo de concentración de políticas públicas. Un punto de inflexión fue la intervención del Programa de Mejoramiento de Barrios (Promeba), mediante el desarrollo del proyecto denominado Grupo Riberas del Salí. En ese marco, de acuerdo a lo establecido en el proyecto Sector Capital Norte-Etapa 1, se llevaron a cabo obras de infraestructura y equipamiento urbano. El barrio fue seleccionado por el Instituto Provincial de Vivienda sobre la base de la magnitud del espacio que ocupa y su cantidad de habitantes (siendo uno de los más populosos entre los asentamientos informales del GSMT) que, además, está localizada en una zona de emergencia ambiental.

Debido a la magnitud que en los años sucesivos alcanzó la problemática del narcomenudeo y el consumo de drogas en el barrio, a mediados de 2012, desde el Ministerio de Desarrollo Social, se inicia una estrategia de abordaje de jóvenes en consumo para la recuperación de las adicciones por consumo de drogas, a partir del trabajo de los DSA. La tarea de los técnicos, de carácter territorial, estuvo articulada con diferentes problemáticas y actores en un barrio caracterizado por una significativa densidad de agentes del Estado y organizaciones sociales, generando confusión y superposiciones de tareas en el territorio. Como resultado de dicha problemática surgen, desde el seno del propio barrio, grupos autoconvocados para contrarrestar el avance del narcotráfico y la problemática del intercambio y consumo de drogas: las Madres del pañuelo negro se conforman a raíz de los numerosos fallecimientos de jóvenes a causa del consumo de paco y posteriormente La hermandad de los barrios, compuesto por vecinos de siete barridas populares de Tucumán, entre ellas, la Costanera.

\section{Radio comunitaria como estrategia terapéutica y fortalecedora de la subjetividad}

Como estrategia de intervención, el DSA promovió la creación de un programa de radio propio, organizado por el grupo de jóvenes en recuperación por consumo de drogas con el que trabajaban.

La experiencia de comunicación popular mediante el programa de radio comenzó en julio de 2013, luego de que el grupo recibiera la oferta de un espacio en una radio clandestina del barrio perteneciente a un vecino. Así fue como, luego de que el grupo de jóvenes analizara y

alimentación, las compras o cualquier otro consumo que sea diagnosticado compulsivo por un profesional de la salud". 
aceptara la propuesta, el DSA contactó a comunicadores de la Agencia de Prensa Alternativa $(\mathrm{APA})^{7}$, solicitando colaboración en el proyecto.

De esta manera se dio inicio a un proceso de trabajo que sostuvo encuentros semanales bajo la modalidad de talleres, en los que APA acompañó al grupo de jóvenes en la organización, armado y producción del programa radial en una primera etapa, dando lugar a jornadas de ensayo y programas pilotos hasta la emisión formal del programa.

El encuadre terapéutico dado por los DSA se retroalimentaba del proceso de revalorización de los discursos y una nueva rutina de actividades que se sostuvo durante un año, en el que el grupo de jóvenes mantuvo la producción y conducción del mismo, al que llamaron Ganas de vivir, nombre que también adoptaron como grupo. Allí transmitieron información inherente al barrio y denunciaron necesidades insatisfechas e injusticias individuales y comunitarias, además de contar sus historias personales en primera persona. El programa salía al aire todos los viernes, de 15 a 17, a través de la frecuencia 103.1, FM el Ángel David y Nélida, una radio clandestina y precaria, propiedad de un vecino que cedió gratuitamente el espacio al grupo de jóvenes.

El programa llegó a su fin imprevistamente hacia fines de 2014, a raíz de un conflicto desencadenado ante el reclamo del dueño de la radio a los DSA, solicitando algún tipo de contribución monetaria a cambio del espacio. Este vecino motivó su petición a partir de una serie de recursos y beneficios (colchones, ladrillos, etc.) que llegaron para algunas familias del barrio (de jóvenes adictos) por parte del Ministerio de Desarrollo Social. Ante la imposibilidad de los técnicos de afrontar el pedido, dado que no manejaban recursos ni decidían sobre la disposición de los mismos, los jóvenes dejaron de asistir a la radio. El dueño de la radio aclaró que su conflicto era con los psicólogos, no así con los jóvenes. No obstante, sin el acompañamiento de los profesionales, el grupo decidió detener la tarea.

\section{Disputa por el espacio púbico: una plaza para recolonizar la salud}

También como parte de las iniciativas grupales identificadas y acompañadas desde los DSA, se desarrolla el proyecto de plaza y cancha de fútbol llamada Julio César Cisneros, Gilada, en homenaje a uno de los jóvenes fallecidos en el barrio a causa del consumo de drogas.

En 2016, los DSA convocan al grupo MHaPa (Mejora del Hábitat Participativo, un servicio técnico de alto nivel del Consejo Nacional de Investigaciones Científicas (STAN-Conicet), que ya trabajaba con los DSA en otros barrios, a fin de colaborar en el diseño participativo de este espacio público. El barrio, a su vez, forma parte de la esfera de proyectos del Programa de Mejoramiento de Barrios (Promeba), que interviene desde 2008 y mantiene una

\footnotetext{
7 La Agencia de Prensa Alternativa (APA) se formó en 2012 como un espacio periodístico y documental, materializado a través de una página web de noticias, con el objetivo de promover la circulación de información a partir de una comunicación alternativa, participativa, mediante procesos contrahegemónicos.
} 
coordinación estrecha con los DSA y el equipo $\mathrm{MHaPa}$, articulando esfuerzos desde el proceso proyectual.

La plaza no estaba incluida en el espacio público contemplado por el plan inicial del Promeba, lo que requirió un ajuste del proyecto urbano. Superado el problema, fue posible emprender instancias de consulta y diseño colectivo.

En primer término, la discusión giró en torno a la exclusividad de la actividad deportiva en torno a la práctica del fútbol que, aun con participación de jóvenes mujeres del barrio, sugiere un uso mayormente masculino. El espacio, sumamente restringido en superficie, forzaba discusiones entre vecinos que priorizaban la cancha llegando casi a eliminar por completo cualquier otra actividad planteada, tales como juegos infantiles y el gimnasio a cielo abierto, propuesto sobre todo por mujeres.

La propuesta presenta la cancha acompañada por una plaza desarrollada de manera vertical, fuera de lo usual, con máximo aprovechamiento de espacio y dando cuenta del proceso de participación colectiva.

\section{Resultados}

Los resultados de la investigación se organizan siguiendo el análisis de variables propias de la participación, de modo tal que se caracterizan: 1. Las distintas fases del proceso histórico de cada caso, 2. Los diferentes actores y roles asumidos en la tarea, a partir del análisis de redes, y 3. La descripción de los ámbitos en los que se desarrolla cada proceso.

\section{Cronología y fases de los casos}

Las fases de participación se consideran desde su momento de gestación, dando cuenta de la situación social de partida, junto a la identificación de aspectos que detentan mayor o menor madurez para desarrollar un ejercicio participativo.

Posteriormente, el proceso en sí mismo se evalúa en función del carácter activo o no de la comunidad en el desarrollo nodal de la experiencia. Por último, se evalúan las condiciones en las que queda la comunidad para el desarrollo de futuros proyectos de manera participativa, en función de la experiencia vivida.

\section{Cuadro 1. Fases}

\begin{tabular}{|l|l|}
\hline RADIO & \multicolumn{2}{|c|}{ PLAZA } \\
\hline HISTORICIDAD \\
\hline \multicolumn{2}{|l|}{ Antecedentes } \\
\hline $\begin{array}{c}\text { El antecedente es la organización del } \\
\text { merendero desde 2012. Esta experiencia sirve } \\
\text { a la conformación del grupo de jóvenes. En el } \\
\text { barrio se contabiliza también la experiencia en } \\
\text { parte participativa, desarrollada desde el el }\end{array}$ & $\begin{array}{c}\text { El antecedente es, para algunos de } \\
\text { lientras que, para la totalidad, su } \\
\text { experia está basada en el proceso } \\
\text { llevado adelante por el Promeba desde }\end{array}$ \\
\hline
\end{tabular}




\begin{tabular}{|c|c|}
\hline Promeba. & 2008. \\
\hline \multicolumn{2}{|l|}{ EL PROCESO } \\
\hline \multicolumn{2}{|l|}{ Génesis del proceso } \\
\hline Año 2013 & Año 2017 \\
\hline \multicolumn{2}{|c|}{$\begin{array}{l}\text { Grupal y comunitario, surgen en reuniones de carácter deliberativo, por lo que se } \\
\text { considera una demanda genuina y priorizada por la comunidad. }\end{array}$} \\
\hline \multicolumn{2}{|l|}{ Proyecto } \\
\hline $\begin{array}{l}\text { La comunidad decide sobre aspectos } \\
\text { centrales: } \\
\text {-Temáticas abordadas en el programa } \\
\text {-Día y horario de emisión } \\
\text {-Musicalización } \\
\text { - Invitados }\end{array}$ & $\begin{array}{l}\text { La comunidad decide sobre } \\
\text { aspectos principales: } \\
\text { - Conservación de la } \\
\text { localización } \\
\text { - } \text {-Actividades que contempla el } \\
\text { proyecto } \\
\text { - - Disposición y diseño } \\
\text { - - Modo de ejecución }\end{array}$ \\
\hline \multicolumn{2}{|l|}{ El proceso } \\
\hline $\begin{array}{l}\text { Producción ajustada a las necesidades. } \\
\text { Emisión óptima de programas al cabo de } \\
\text { un año. } \\
\text { Abrupto final debido a problemas ajenos a } \\
\text { los jóvenes. } \\
\text { Final asociado a la frustración en cuanto a } \\
\text { experiencias comunicacionales }\end{array}$ & $\begin{array}{l}\text { Diseño apropiado. } \\
\text { La gestión por los recursos se lleva } \\
\text { adelante -infructuosamente- desde los } \\
\text { técnicos DSA y Promeba. } \\
\text { La cancha se utiliza aun sin } \\
\text { completarse el proyecto, por lo que se } \\
\text { trata de un espacio sin cualificación } \\
\text { (proyecto de ejecución inconclusa). }\end{array}$ \\
\hline \multicolumn{2}{|l|}{ Tendencia } \\
\hline Finaliza en 2014. & Decae en 2018. \\
\hline $\begin{array}{l}\text { Frustración. Baja expectativa y confianza en } \\
\text { érdida de los recursos materiales para dar cor }\end{array}$ & $\begin{array}{l}\text { ocesos de este tipo debido a la falta o } \\
\text { luidad a la actividad. }\end{array}$ \\
\hline
\end{tabular}

Estas formas de participación se adecuaron a las necesidades y posibilidades de los jóvenes, otorgando -en el caso radial- valor a su palabra a partir del relato de historias en primera persona, mediante la construcción de procesos de identificación con los oyentes. No obstante, cabe destacar que la frustración también forma parte del mismo proceso, a raíz de conflictos ajenos a los jóvenes, dificultando nuevos espacios comunicacionales. El derrotero sistematizado da cuenta de un progreso madurativo para algunos jóvenes y vecinos del barrio que han participado en ambas experiencias. Este análisis incluye a los técnicos de los DSA que desarrollan cada vez más actividades de diferente naturaleza, apoyados en otros técnicos especializados que comparten su lógica de trabajo. No obstante, para las dos 
experiencias, la falta de apoyo institucional en términos de recursos -aun con la presencia de técnicos provinciales- resulta determinante para la discontinuidad de los procesos. Este antecedente representa un retroceso sobre el objetivo de fortalecimiento comunitario a partir del desarrollo de procesos participativos.

\section{Red de relaciones y vínculos}

Cada experiencia se analiza recurriendo al análisis de redes que permite la visibilización de la red de relaciones que operan sobre un proceso específico, dando cuenta de la construcción o no de vínculos imprescindibles para el desarrollo de participación.

En el caso de la experiencia de comunicación popular mediante el proyecto del programa de radio comunitaria, tuvo un rol protagónico por parte de los jóvenes en recuperación del consumo de drogas, con el encuadre terapéutico del DSA y la colaboración de los comunicadores de APA. Este trinomio constituye la base vincular que habilita el desarrollo de la actividad.

El vecino, dueño de la radio, también asume un papel fundamental durante el proceso, al ser el facilitador del espacio radial, como así también de su interrupción. Durante el periodo en el que el programa salió al aire, los jóvenes contaron con la participación de una serie de invitados quienes, a partir de la producción de APA, respondían a la convocatoria y visitaban la radio en el barrio para dialogar sobre distintas problemáticas. La radio recibió apoyo de diversos referentes barriales y académicos, tales como La hermandad de los barrios y las Madres del pañuelo negro, así como profesores universitarios que en ocasiones visitaban el programa.

\section{Esquema 1. Análisis de redes sobre proyecto de programa radial comunitario}

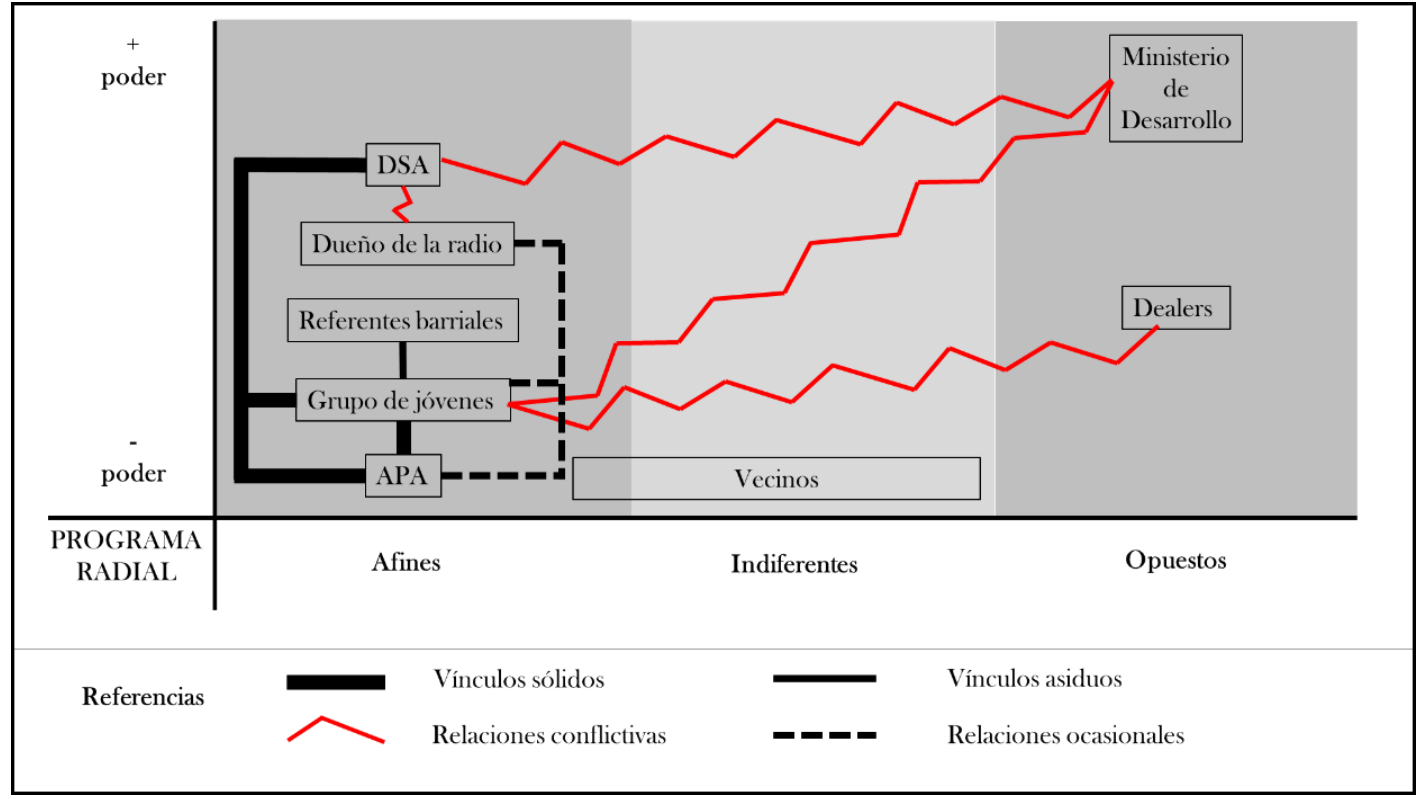


Fuente: elaboración propia a partir del trabajo de campo.

Entre todos los actores intervinientes durante el desarrollo del proyecto de radio, los vínculos más sólidos se desenvolvieron entre los jóvenes en recuperación del consumo de drogas, sus familias y el DSA. Esto constituye un resultado esperado a partir de la metodología implementada en el trabajo del abordaje terapéutico, dividido en tres niveles de intervención: individual, grupal y comunitario. Individual, a partir del seguimiento y acompañamiento terapéutico de algunos de los jóvenes en cuanto a su problemática de consumo, lo que implicaba concurrir a sus viviendas y realizar entrevistas analizando el contexto individual, la historia familiar-personal y la situación de consumo. Grupal, mediante la participación en el espacio terapéutico de reunión semanal. Y comunitario, a partir de la organización y participación en jornadas de trabajo y festejos, mediante la articulación con otros actores. Del mismo modo, a raíz del proceso de trabajo semanal sobre el proyecto de radio, los jóvenes del grupo, especialmente quienes participaron en forma activa del programa -conductores, columnistas-, desarrollaron vínculos sólidos con los comunicadores de APA, del mismo modo sucedió con el DSA. En cuanto al dueño de la radio, se presentaron relaciones distintas según los actores con los que interactuó. Con los jóvenes del grupo y con los comunicadores de APA, quienes visitaban la radio semanalmente para la emisión del programa, se logró una buena relación basada en encuentros ocasionales. No obstante, si bien al inicio del proceso mantuvo una buena relación con el DSA, luego comenzó a desarrollarse una relación conflictiva, a raíz de los nuevos intereses personales y económicos del vecino, que concluyó con el final abrupto del programa. Por otro lado, dada la naturaleza de los temas abordados por los jóvenes en el programa, problemáticas derivadas principalmente a causa de la venta y el consumo de drogas, estos mantenían una relación de conflicto con aquellos vecinos relacionados al narcomenudeo. Del mismo modo, la mayoría de las veces la indiferencia de algunos vecinos se debía al temor que estos padecían debido a los temas tratados en el programa y a posibles represalias de los comercializadores de droga.

En cuanto al Ministerio de Desarrollo Social y sus funcionarios, mantuvieron relaciones conflictivas con la comunidad, debido al público conocimiento sobre el sostenimiento de una precaria condición laboral hacia los técnicos del DSA. Esto dificultaba ostensiblemente su tarea en el territorio, negando el respaldo sobre las actividades que se desplegaban a partir de su tarea, como el caso de la radio. La situación particular de los DSA se suma a la falta de cumplimiento de compromisos de diferente orden convenidos desde otras áreas del mismo ministerio para la resolución de problemáticas barriales de diferentes tipos.

En el caso de la experiencia de mejora del hábitat a partir de la recualificación del espacio público, intervinieron diferentes actores, además de los DSA y las familias de jóvenes en proceso de tratamiento. Nuevos referentes políticos, identificados como emisarios de 
diferentes funcionarios de gobierno y también de la Universidad y el Conicet, como se mencionó anteriormente, a través del grupo $\mathrm{MHaPa}$, en estrecha articulación con el equipo técnico del Promeba.

Se presentan también organizaciones como La hermandad de los barrios que incorpora entre sus reivindicaciones a la mejora del hábitat, comprendiendo su relevancia en la problemática de la venta y el consumo de drogas. Los integrantes de esta hermandad provienen de distintos barrios populares de la provincia de Tucumán, donde se destacan algunos referentes del barrio Costanera Norte.

\section{Esquema 2. Análisis de redes sobre proyecto de mejora del hábitat}

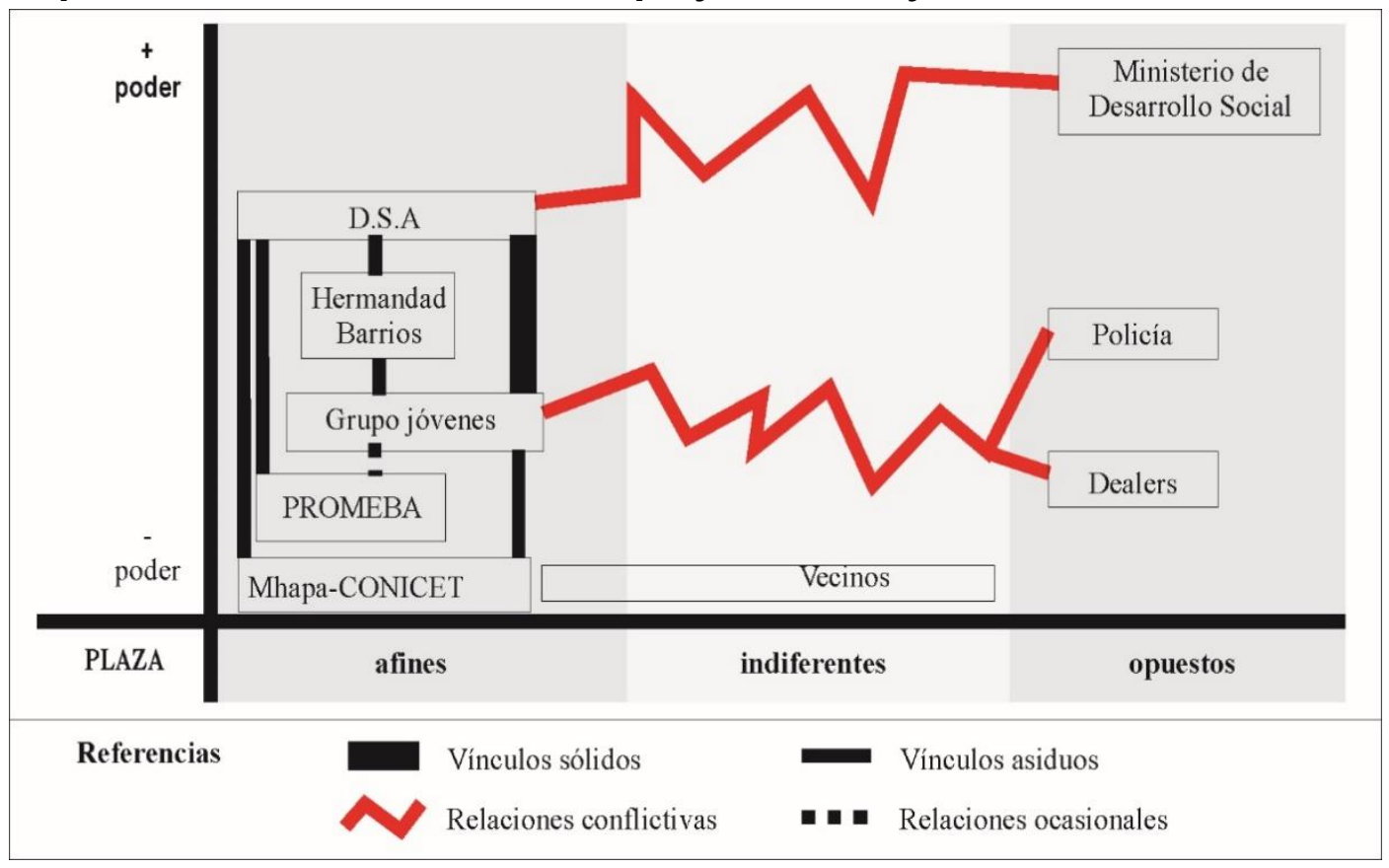

Fuente: elaboración propia a partir del trabajo de campo.

Nuevamente, los principales vínculos construidos se desarrollan entre las familias y los DSA, a partir de la tarea principal que es terapéutica. Es esta necesidad el motor fundante de la tarea, la que abre nuevas necesidades complementarias y vínculos con los que se establecen acuerdos a partir de otros aspectos como la mejora del hábitat, considerado un emergente directo entre las múltiples necesidades irresueltas dadas en el contexto general de vulnerabilidad que sobrelleva la población del barrio. En esa relación se incorpora de manera armónica y complementaria el equipo técnico del Mhapa, que lleva adelante la coordinación del proceso proyectual; a partir de pautas dispuestas por el DSA, combinadas 
con su estrategia de participativa de diseño, ambas compatibles, desde donde se establecen las actividades y se cumple el primer objetivo, acordar un proyecto.

De esta forma y a modo de síntesis, se presentan los aspectos clave constituyentes de los vínculos desarrollados en cada experiencia.

\section{Cuadro 2. Vínculos de cada experiencia de estudio}

\begin{tabular}{|c|c|}
\hline RADIO & PLAZA \\
\hline \multicolumn{2}{|l|}{ En cuanto a la constitución de roles } \\
\hline \multicolumn{2}{|c|}{$\begin{array}{l}\text { Los DSA se desempeñan como promotores y coordinadores de los procesos en ambos } \\
\text { casos. Predomina su rol terapéutico. }\end{array}$} \\
\hline $\begin{array}{l}\text { Grupo de jóvenes: rol activo en la } \\
\text { producción y conducción del programa. }\end{array}$ & $\begin{array}{l}\text { Grupo de jóvenes y sus familias, sobre } \\
\text { todo mujeres, asumen las principales } \\
\text { responsabilidades. }\end{array}$ \\
\hline $\begin{array}{l}\text { Referentes barriales: acompañan } \\
\text { respondiendo a la invitación de los jóvenes } \\
\text { al programa. }\end{array}$ & $\begin{array}{l}\text { Referentes barriales: incorporan las } \\
\text { mejoras de hábitat entre sus } \\
\text { reivindicaciones. }\end{array}$ \\
\hline $\begin{array}{l}\text { Dueño de la radio: facilita y luego } \\
\text { restringe el uso del espacio radial. }\end{array}$ & $\begin{array}{l}\text { Punteros políticos. Tratan de apropiarse } \\
\text { del espacio. No logran prevalecer. }\end{array}$ \\
\hline $\begin{array}{c}\text { APA, facilitadores del proceso de } \\
\text { producción del programa radial. }\end{array}$ & $\begin{array}{l}\text { MHaPa, técnicos abocados al proceso } \\
\text { proyectual. }\end{array}$ \\
\hline $\begin{array}{l}\text { Acompañamiento de la emisión del } \\
\text { programa a lo largo de todo el periodo en } \\
\text { el que salió al aire. }\end{array}$ & $\begin{array}{l}\text { Promeba, técnicos facilitadores de } \\
\text { recursos y coordinación con el proyecto de } \\
\text { regularización y mejora macro. }\end{array}$ \\
\hline \multicolumn{2}{|l|}{ Relaciones intercomunitarias } \\
\hline $\begin{array}{l}\text { La comunidad presenta condiciones de } \\
\text { integración. Dada la participación de los } \\
\text { jóvenes del grupo en el proceso de } \\
\text { comunicación comunitaria, sus familias } \\
\text { resultan involucradas. Del mismo modo, } \\
\text { con el tiempo se logra el interés de gran } \\
\text { parte de amigos y vecinos. }\end{array}$ & $\begin{array}{l}\text { La comunidad presenta condiciones de } \\
\text { integración entre las familias que llevan } \\
\text { adelante la experiencia. En un contexto } \\
\text { general de deterioro en los vínculos } \\
\text { vecinales, donde el espacio público que } \\
\text { representa un ámbito de peligro. }\end{array}$ \\
\hline $\begin{array}{l}\text { El desarrollo del proceso evidenció el } \\
\text { flujo del poder en la realización de la tarea } \\
\text { por parte de los actores intervinientes. No } \\
\text { obstante, hacia el final logró imponerse la } \\
\text { concentración de poder por parte del } \\
\text { dueño de la radio. }\end{array}$ & $\begin{array}{l}\text { Durante el proceso se repartió el flujo de } \\
\text { poder y toma de decisiones; no obstante, } \\
\text { existe dependencia de la iniciativa de los } \\
\text { Dispositivos de Salud para lograr avances. }\end{array}$ \\
\hline \multicolumn{2}{|c|}{ Relaciones extracomunitarias (comunidad-institución) } \\
\hline
\end{tabular}


Predomina el conflicto. Debido a la falta de respuestas por parte de las instituciones del Estado, específicamente del Ministerio de Desarrollo Social, hacia las necesidades y problemáticas de la comunidad. Esto ocurre aun cuando los técnicos de estas instituciones motorizan las actividades sobre las que se espera el respaldo.

\begin{tabular}{l|l}
- & \multicolumn{1}{|c}{ El Promeba no logra romper con su } \\
estructura cerrada de implementación e \\
incorporar plenamente este proyecto para \\
materializarlo.
\end{tabular}

\section{Objetivos de los procesos de mejora barrial}

Objetivo general compartido: recuperación de jóvenes adictos mediante acciones individuales y grupales, a partir de procesos de resolución participativa de necesidades consideradas prioritarias.

Objetivo particular: fortalecer las subjetividades a partir de la revalorización de sus discursos.

Dinamizar un proceso de comunicación alternativa desde el propio barrio.

Ambos confluyentes con el objetivo general.

\section{Proceso re-politizante}

\section{Disponibilidad y la disposición en las tareas de mejoramiento barrial}

DSA son el principal recurso público facilitado para esta tarea. No obstante, se trata de pocos profesionales (3) y en condiciones de trabajo precarizado. De esta forma, si bien existe disponibilidad, es escasa. Su disposición, por el contrario, es elevada y evidencia un significativo compromiso individual y grupal de los integrantes del equipo técnico para el desarrollo de la tarea.

Jóvenes del grupo, abocados a las Jóvenes en recuperación con sus familias. actividades derivadas del proceso de Fundamentalmente mujeres, destinan recuperación, en ocasiones se registran momentos de menor interés y participación, aunque la actividad se sostiene regularmente durante el año de trabajo. Esto hace referencia a buenas condiciones de disposición, aunque su disponibilidad depende de los espacios de tiempo que les deja el trabajo informal.

APA. Tiempo de trabajo organizado en una jornada semanal de encuentro y tarea. tiempo de su cotidianeidad al trabajo vecinal. Por lo tanto, su disponibilidad no tiene ningún tipo de recurso facilitador. Es su compromiso con la tarea lo que justifica su disposición a trabajar en los proyectos de este proceso.

Mhapa. Ocupan tiempo de trabajo acotado, su principal tarea es investigación 


\section{Su principal tarea es de acompañamiento al grupo, que se desarrolla de manera gratuita. Es por eso que se destaca el compromiso y disposición hacia el proyecto, ya que su disponibilidad depende del desarrollo de trabajos remunerados en otros espacios.}

\section{y asumen este proyecto como labor} complementaria, por lo que tienen una disponibilidad reducida. Su tarea se desarrolla de manera gratuita y contribuyen con elevada disposición en los aspectos inherentes a la producción del hábitat.

Promeba. Se trata de pocos profesionales (2), precarizados y sobre-atareados, por lo que a pesar de tener cierta disponibilidad, se destaca su disposición a resolver necesidades que superan en magnitud la composición de su equipo de trabajo.

\section{Obstáculos epistemológicos y epistemofílicos}

La solidez metodológica de los DSA permite un encuadre apropiado a la totalidad del proceso, ya que sobre su lógica y estrategias se ordenan ambas actividades.

El grupo de comunicadores de APA El grupo Mhapa presenta una formación desarrolla para esta experiencia una y estrategia metodológica específica, modalidad de trabajo apropiada, dando orientada ya previamente a este tipo de cuenta de una estrategia habilitante que actividades, por lo que se desarrolla en incluso contribuye a fortalecer su grupo. forma apropiada y consolida su relación con Ios DSA.

\section{Comunicación y aprendizaje}

Flujo continuo de información y aprendizaje.

El conocimiento sobre las teorías que sustentan la comunicación, así como el manejo operativo de una radio, fortalecen el aprendizaje del uso de herramientas y potencian discursos comunitarios para la integración social.
El conocimiento sobre el proceso proyectual se encuentra adaptado de manera tal de entrelazar a los protagonistas barriales con los instrumentos de diseño aportados por los técnicos. Esto se contrapone a procesos de producción de hábitat tradicionales en los que la comunidad es espectadora en el proceso de diseño.

Como síntesis, cabe explicitar la importancia de la solidez metodológica de los DSA para el proceso general y los técnicos de carácter complementario como APA, Mhapa y Promeba, que permiten que la estrategia terapéutica despliegue nuevos matices sobre aspectos que los DSA no lograrían resolver solos. Las relaciones entre ellos y con la comunidad son 
dialógicas, de manera tal que, si los recursos hubieran sido puestos a disposición, es posible que ambas experiencias tuvieran continuidad y nuevos ribetes de desarrollo transformadores y multiplicadores del objetivo terapéutico principalmente, y otros complementarios que surgen en el momento y bajo el modo en que la comunidad tiene la posibilidad de identificar y emprender la resolución de nuevas problemáticas.

Este aspecto evidencia nuevas formas de promoción de la reconstrucción del tejido social, bajo los nuevos términos que plantea el contexto de deterioro social actual. Dichas articulaciones si bien no son nuevas, son inéditas al momento de confluir en la búsqueda de promoción de salud en un sentido ampliado. Salud como capacidad de transformación y producción de proyectos, como horizonte dotado de expectativas que contribuyen a revertir la frustración, originada en el desequilibrio entre necesidad y satisfacción constante en el contexto de pobreza dado.

\section{Los ámbitos de la participación}

Este apartado recupera las experiencias de construcción de espacios de participación según cada caso, a partir del establecimiento de distintas modalidades de trabajo que los han caracterizado. Se trata de distintas dinámicas adoptadas en diferentes etapas de los procesos por parte de los actores intervinientes.

\section{Cuadro 3. Ámbitos de trabajo}

\begin{tabular}{|c|c|}
\hline CASO AUTOGESTIONADO & CASO ESTATAL \\
\hline \multicolumn{2}{|l|}{ Ámbito deliberativo/asambleario } \\
\hline \multicolumn{2}{|c|}{$\begin{array}{l}\text { Este espacio en ambos casos está dado por el espacio terapéutico. Es aquí donde se } \\
\text { definen los proyectos complementarios como la radio y la plaza, con participación } \\
\text { protagónica de la comunidad al momento de elegir y definir nuevos proyectos y } \\
\text { estrategias de acción. }\end{array}$} \\
\hline \multicolumn{2}{|l|}{ Movilizaciones } \\
\hline \multicolumn{2}{|c|}{$\begin{array}{l}\text { Se desarrollan a partir de la participación en organizaciones como La hermandad de } \\
\text { los barrios, bajo la demanda de mayor presupuesto para la atención terapéutica } \\
\text { mediante el aumento de DSA y acceso a recursos para sostener los proyectos que } \\
\text { surgen de allí. }\end{array}$} \\
\hline \multicolumn{2}{|l|}{ Gestiones } \\
\hline $\begin{array}{l}\text { La gestión se realiza a partir de la } \\
\text { palabra de representantes rotativos, } \\
\text { acompañados por técnicos. }\end{array}$ & $\begin{array}{l}\text { La gestión se realiza dentro del propio } \\
\text { barrio, por el elevado nivel de presencia } \\
\text { institucional. }\end{array}$ \\
\hline \multicolumn{2}{|l|}{ Talleres } \\
\hline $\begin{array}{l}\text { Actividad destinada al armado del } \\
\text { proyecto radial. 1. Los primeros encuentros }\end{array}$ & $\begin{array}{l}\text { Actividad destinada al armado del } \\
\text { proyecto. Se desarrolla una secuencia de: }\end{array}$ \\
\hline
\end{tabular}


son destinados a la discusión en torno a la necesidad grupal de un programa radial: qué, cómo, porqué, para quién. 2. Posteriormente se pone en común cuestiones técnicas y conceptuales. 3. Seguido de ensayos y pruebas pilotos.

1. Demanda general y programa de
necesidades, 2 . Presentación de
alternativas y selección de aspectos
positivos de cada una, 3. Presentación de
propuesta final para iniciar el proceso de
gestión.
gestión.

\section{Relevamientos}

Se realizan de parte del Promeba en cuanto a las condiciones sociohabitacionales. Los DSA recorren el barrio y registran información territorial sobre consumo, intercambio de drogas, problemas de salud relacionadas a la adicción, historias familiares, datos socioeconómicos, conflictos vecinales, etc.

Los ámbitos de trabajo están estructurados a partir de la tarea terapéutica, liderada también por los DSA que coordinan y monitorean el proceso general y los subproyectos que se desarrollan con este encuadre. Esta coordinación general resulta vital, ya que las condiciones particulares que atraviesan los principales destinatarios del proceso, jóvenes consumidores en proceso de recuperación, tratan una condición delicada cuya naturaleza resulta compleja para técnicos que no están formados específicamente sobre esta problemática.

\section{Conclusiones}

El contexto en el que se desarrollan las experiencias es particularmente adverso, ya que las condiciones sociales requeridas para un proceso participativo pleno demandan cierto grado de integración social de partida y madurez en el establecimiento de relaciones habilitantes para esta dinámica, tanto intra como extracomunitarias. No obstante, el barrio Costanera Norte presenta elevados niveles de violencia y fragmentación comunitaria. Es en este contexto que las experiencias dan cuenta de haber atravesado procesos participativos, evidenciando estrategias y transformaciones en dos niveles: 1 . Sobre el aspecto central que es el terapéutico, en tanto promovió la salud en el proceso de recuperación del consumo de drogas para los jóvenes que participaron activamente; y 2 . Sobre la comunicación y el hábitat, respectivamente.

La experiencial radial contribuye a un proceso de democratización de la comunicación, en tanto promovió la circulación de la información desde dentro del barrio hacia afuera del mismo, disputando los discursos dominantes de los medios hegemónicos de la provincia. Dicho proceso superó los límites tecnocéntricos, más allá de la herramienta concreta de la radio, convirtiéndose en una experiencia de comunicación territorial de base, dentro de un proceso más general de resistencia. Redefiniendo la palabra-pensamiento, como conquista y derecho inalienable, reforzando aspectos de su propia identidad lingüística, cognoscitiva, 
afectiva, política y sociocultural (Requejo, 2009). A partir de la ruptura del cerco mediático, se constituyó como un proceso desestigmatizador que disputó el sentido otorgado al barrio Costanera Norte para la opinión pública. La disputa de sentidos se traslada hacia los límites de la hegemonía, desplazando las fronteras de lo decible. Se asume un posicionamiento subjetivo e ideológico activo, así como de interpretación crítica de la realidad, que implicó la resignificación del rol de los jóvenes con problemas de consumo dentro del barrio y hacia afuera, poniendo en valor los aspectos saludables. Se ejerce su derecho a ser autores y productores de conocimiento e información, y no meramente usuarios, receptores o reproductores. Sujetos protagonistas de un proceso contrahegemónico, quienes disputan un lugar en los circuitos de comunicación de los sectores de poder, al imponer en la agenda mediática la cotidianeidad del barrio desde su autonomía igualitaria. Se redefinen de este modo las relaciones de poder, invirtiendo los sentidos estigmatizadores, a partir del reconocimiento de sus heterogeneidades.

El proceso de mejora del hábitat a partir del proyecto sobre un espacio público se desarrolla en forma plena y con una potencia que logra revertir el uso nocivo del espacio durante la experiencia. Aun cuando resulta dificultoso recaracterizar el espacio en forma aislada, la mejora lenta pero integral llevada adelante desde el Promeba genera condiciones alentadoras para el progreso de esta suerte de recolonización del espacio público.

Cuando se esperaba que los obstáculos fueran de orden metodológico por la complejidad particular del grupo humano comprometido, finalmente el principal obstáculo fue la falta de apoyo institucional, que devela los verdaderos intereses de las esferas de poder gubernamental.

Para terminar, entendemos que la práctica participativa es compatible y recomendable -e imprescindible- para el abordaje terapéutico de tipo territorial planteado desde las políticas de prevención y tratamiento de adicciones al consumo de drogas. En el caso de los sectores populares, la dinámica de consumo e intercambio de drogas se encuentra estrechamente vinculada a la situación de vulnerabilidad, amparada por la imposibilidad de construir una perspectiva futura individual, grupal y colectiva, configurando un retroceso cualitativo que puede revertirse -entre otros mecanismos- con la apuesta real a la participación.

\section{Bibliografía}

Arrieta, E.M.; Prieto, J. y Damin, C. (2020). Paco o pasta base. En Arrieta, E.M. (comp.) Un libro sobre drogas (pp. 237-243). Buenos Aires: El Gato y La Caja.

Arroyo, S. (2007). Activos, suelo urbano y hábitat popular desde la perspectiva de la Economía Social. Estudios de caso en la Ciudad de Buenos Aires. Tesis de maestría. Buenos Aires, Argentina.

Boldrini, P. (2011). Producción participativa del hábitat popular en el área metropolitana de Tucumán. Tesis de doctorado. Tucumán, Argentina: Facultad de Filosofía y Letras UNT. 
(2019). Adicciones y narcomenudeo, barreras (in) franqueables para los asentamientos informales en Tucumán, Argentina. En el marco del I Encuentro de la Red de Asentamientos populares. Córdoba, Argentina.

(Sin fecha). Participación popular en la producción del hábitat. El caso del Área Metropolitana de Tucumán-Argentina. Revista Norte Grande. (En prensa).

Bolsi, A. y Paolasso, P. (comp.) (2009). Geografía de la pobreza en el Norte Grande Argentino ( $1^{\mathrm{a}}$ ed.) Tucumán, Argentina: UNT-Conicet.

Borja, J. y Muxi, Z. (2000). El espacio público, ciudad y ciudadanía. Barcelona.

Correal Espina, G. (2006). De la planificación al ordenamiento del territorio. En Moncaleano Archila, A. (comp.) Hábitat y el derecho a la vivienda digna. Bogotá: La Imprenta Ed. Ltda.

Cravino, M.C. y Varela, O.D. (2008). Los mil barrios (in)formales en el AMBA. Los Polvorines: UNGS.

Damin, C y Arrieta, E. M. (2020). ¿Qué es la reducción de riesgo y daños? En Arrieta E.M. (comp.) Un libro sobre drogas (pp. 276-291). Buenos Aires: El Gato y La Caja.

De Moraes, D. (2011). La cruzada de los medios en América Latina: gobiernos progresistas y políticas de comunicación. Buenos Aires: Ediciones Paidós.

De Moraes, D.; Ramonet, I. y Serrano, P. (2013). Medios, poder y contrapoder. De la concentración monopólica a la democratización de la información. Buenos Aires: Biblos.

Del Castillo, A. (2012). Pobreza y 'cartoneo' en un barrio periférico del Gran San Miguel de Tucumán. Cuadernos. FHyCS-UNJu, 41, 249-271.

Del Frade, C. (2014). Ciudad blanca, crónica negra. Postales del narcotráfico en el Gran Rosario, Santa Fe, Córdoba y Buenos Aires. Santa Fe, Argentina: Editorial Último Recurso.

Fals Borda, O. (1986). La investigación-acción participativa: Política y epistemología. En Álvaro Camacho, G. (ed.) La Colombia de hoy (pp. 21-38). Bogotá: Cerec.

Fernández, E. y Smolka, M.O. (2004). Regularización de la tierra y programas de mejoramiento: nuevas consideraciones. Land Lines, (16)3.

Glaser, B. y Strauss, A. (1967). The discovery of grounded theory. Chicago: Aldine.

Gómez López, C. (2000). Influencia de la Promoción Pública de Vivienda en el Desarrollo de la Mancha Urbana de la Ciudad de San Miguel de Tucumán. Elementos para la Comprensión de su Estructura Urbana. Tesis doctoral. Valencia.

Gómez López, C.; Cuozzo; Boldrini, P. (2012). Nuevas lógicas de expansión en la dinámica urbana de una ciudad intermedia. El caso del aglomerado tucumano en el norte argentino. En el marco del Coloquio de Transformaciones Territoriales, Grupo de universidades Montevideo. Tucumán, Argentina.

Gómez López, R. (1976). Asentamientos marginales de vivienda en San Miguel de Tucumán. Tucumán, Argentina: Facultad de Arquitectura y Urbanismo, UNT. 
Grimson, A. (2011). Los límites de la cultura. Críticas de las teorías de la identidad. Buenos Aires: Siglo Veintiuno Editores.

Guber, R. (2009). El salvaje metropolitano. Buenos Aires, Argentina: Legasa.

Hammersley, M. y Atkinson, P. (1994). Etnografía. Métodos de investigación (1a ed.) Barcelona: Ediciones Paidós.

Harvey, D. (2007). Espacios del Capital, hacia una geografía crítica. Madrid: Ediciones Akal.

Hernández García, J. (2005). Participación y hábitat: ¿Sueño posible o relación no deseada? Revista INVI, 55. Chile: Universidad de Chile.

Jiménez Domínguez, B. (comp.) (2008). Subjetividad, participación e intervención comunitaria, una visión lógica desde América Latina ( $1^{\mathrm{a}}$ ed.) Argentina: Editorial Paidós.

Llovera M. S. y Scialla M. (2016). La de-construcción del problema droga: ¿nuevas soluciones para viejos problemas? En III Jornadas de Estudios de América Latina y el Caribe (IELAC). CLACSO - UBA, Buenos Aires, Argentina.

Llovera, M., y Scialla, M. (2017). Políticas de drogas en Argentina (2003-2015). Reflexiones en torno a la puja entre lo heredado y lo reformulado. Temas y Debates (34): 77-99.

Malizia, M. y Boldrini, P. (Sin fecha). Las lógicas de ocupación del espacio urbano. Un estudio de realidades contrapuestas. El caso de las urbanizaciones cerradas y villas miseria en yerba buena, Gran San Miguel de Tucumán. Revista Cuadernos (en prensa).

Malizia, M.; Boldrini, P. y Paolasso, P. (2018). Hacia otra ciudad posible. Transformaciones urbanas en el aglomerado Gran San Miguel de Tucumán. Buenos Aires: Editorial Café de las Ciudades.

Marafioti, R. (2010). Sentidos de la comunicación. Teorías y perspectivas sobre cultura y comunicación. Buenos Aires: Editorial Biblos.

Mercadé, F. (1986). Metodología cualitativa e historias de vida. Revista Internacional de Sociología, 44(3):295-320.

Mustafá, E. (2016). El narcotráfico como un factor de violencia, fragmentación y degradación vincular en nuestra sociedad [en línea]. Recuperado de http://apaprensa.com.ar.

Ortiz Flores, E. (2008). La producción social del hábitat [en línea]. Consultado el 20 de octubre de 2009. Recuperado de http://www.moi.org.ar.

Paolasso, P. (2004). Los cambios en la distribución espacial de la población en la provincia de Tucumán durante el siglo XX. Tesis de doctorado. Facultad de Filosofía y Letras, UNT.

Paolasso, P.; Malizia, M. y Longhi, F. (2011). Vulnerabilidad y segregación socioespacial en el Gran San Miguel de Tucumán. En Vergara Durán, A. (comp.) Vulnerabilidad en grandes ciudades de América Latina (pp. 50-71). Colombia: Editorial Unicorte.

Pelli, V.S. (1995). Pobreza Habitacional e Iniciativas Gubernamentales y no Gubernamentales. En el marco del Seminario Internacional Política Habitacional en Argentina, Reestructuración Global y Desarrollo Sustentable. Mesa Redonda, Buenos Aires. 
Picas Contreras, J. (2001). El papel de las organizaciones no gubernamentales y la crisis del desarrollo una crítica antropológica a las formas de cooperación. Tesis doctoral. Programa de doctorado de Antropología Social Bienio 1994-1996, Facultat de Geografia i Història Universitat de Barcelona. Disponible en http://www.tdx.cat/TDX-0630106-140250.

Pichon-Rivière, E. y Pampliega de Quiroga, A. (2009). Psicología de la vida cotidiana. Buenos Aires: Nueva Visión.

Quiroga, A. (2001). Enfoques y perspectivas en psicología social; desarrollos a partir del pensamiento de Enrique Pichón Rivière. Buenos Aires: Cinco.

Quiroga, A.P. (2005). Crisis y procesos sociales, sujeto y grupo. Desarrollos de la psicología social a partir del pensamiento de Enrique Pichon-Rievère. Buenos Aires: Cinco.

Quiroga, A.P. y Racedo, J. (1995). Crítica a la vida Cotidiana. Buenos Aires: Cinco.

Requejo, M. (2009). Lingüística social y autoría de la palabra y el pensamiento: temas de debate en psicología social y educación. Buenos Aires: Ediciones Cinco.

Rolnik, R. (2009). La democracia en el filo de la navaja: límites y posibilidades para la implementación de una agenda de reforma urbana en Brasil. Revista EURE (Santiago) [online], (35)104, 5-28.

Sabatini, F. (2003). La segregación social del espacio en las ciudades de América Latina, Documentos del Instituto de Estudios Urbanos y Territoriales. Serie Azul No 35. Chile: Pontificia Universidad Católica de Chile.

Sedronar (2010). Estudio Nacional en población de 12 a 65 años, sobre Consumo de Sustancias Psicoactivas. Argentina.

(2017a). Estudio nacional en población de 12 a 65 años sobre consumo de sustancias psicoactivas. Resultados principales de la provincia de Tucumán. Argentina.

(2017b). Estudio Nacional en población de 12 a 65 años, sobre Consumo de Sustancias Psicoactivas. Argentina.

Taylor, S. y Bodgan, R. (1984). Introducción a los métodos de investigación cualitativa. Barcelona, España: Editorial Paidós.

(1990). Introducción a los métodos cualitativos de investigación. Barcelona, España: Editorial Paidós.

Touzé G. (2006). Evolución del 'problema droga' en la Argentina. En: Touzé G. (Org.) Saberes y prácticas sobre drogas: el caso de la pasta base de cocaína (pp. 47-76). Buenos Aires, Argentina: Intercambios.

Trufo M., Brescia F. Píngaro Lefevre C. (2017) Aguantamos contra el Estado, perdemos contra las bandas. Reflexiones sobre la circulación de violencias en tomas de tierras y asentamientos de la Región Metropolitana de Buenos Aires. Quid 16 (6):146-168.

Vasilachis de Gialdino, I. (comp.) (2015). Estrategias de investigación cualitativa. Barcelona, España: Editorial Gedisa. 
Vinelli, N. (2014). La televisión desde abajo. Historia, alternatividad y periodismo de contrainformación. Buenos Aires, Argentina: Colectivo El Topo Blindado. Cooperativa El Río Suena.

Yuni, J.A. y Urbano, C.A. (2003). Recursos metodológicos para la preparación de proyectos de investigación. Volumen II. Córdoba, Argentina: Editorial Brujas 